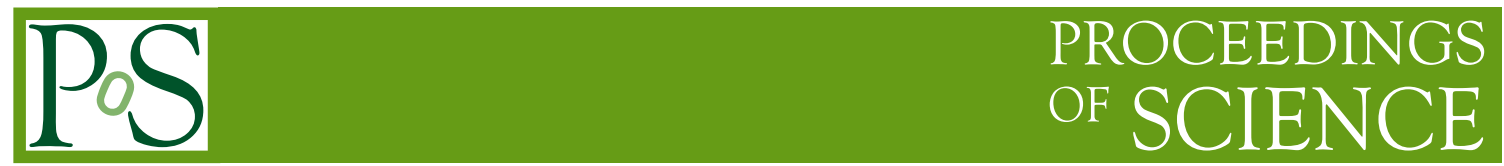

\title{
LHCb Exotica and Higgs searches
}

\author{
Donatella Lucchesi*† \\ University and INFN of Padova \\ E-mail: donatella.lucchesiepd.infn.it
}

LHCb Collaboration has the unique opportunity to search for Higgs production and new physics in regions not accessible by the other LHC experiments. The latest results obtained by exploiting final states with $b$ and $c$ jets with or without an isolated lepton are presented.

38th International Conference on High Energy Physics

3-10 August 2016

Chicago, USA

*Speaker.

${ }^{\dagger}$ On behalf of the LHCb Collaboration 


\section{Introduction}

LHCb uses jets containing $b$ and $c$ quarks to test the standard model (SM), probe the Higgs sector and search for new physics (NP). The Higgs decays $H^{0} \rightarrow b \bar{b}$ and $H^{0} \rightarrow c \bar{c}$ are reconstructed through jets tagged as containing heavy flavor particles. In addition, several NP models predict the production of massive long lived particles (LLP) with decay product which lead to displaced jets containing $b$ quarks. Examples of such particles are the lightest supersymmetric particle in SUSY models [1] and the neutral $\pi_{v}$ [2] particle in hidden valley (HV) models. In these searches the LHCb experiment covers a parameter space complementary to ATLAS and CMS.

The $\mathrm{LHCb}$ detector, described in detail in [3,4], allows the reconstruction of jets in the forward region $2.2 \leq \eta \leq 4.2$. The particle flow algorithm combines together tracking and calorimetry information used by FastJet to form jets based on the anti- $k_{t}$ method with radius $R=0.5$. The heavy flavor content is determined by searching for a secondary vertex (SV) inside the jet cone and by using a Boost Decision Tree (BDT) technique to separate $b$ from $c$ and light quark contribution. The efficiency to tag a $b(c)$ jet goes from $30 \%(10 \%)$ up to $65 \%(25 \%)$ as the mistag probability for a light-parton jet goes from almost $0 \%$ to at maximum $0.5 \%$ [5].

\section{Search for the SM Higgs boson decaying to a $b \bar{b}$ or $c \bar{c}$ pair}

While CMS and ATLAS [6] have measured $H \rightarrow b \bar{b}$ production in association with a vector boson, the combined statistical significance is only $2.6 \sigma$. The observed signal has a statistical significance of $2.6 \sigma$, which demonstrates the difficulty of reconstructing this decay channel in a hadronic environment.

LHCb uses a sample of events collected at $\sqrt{s}=8 \mathrm{TeV}$ by requiring a high momentum electron $\left(P_{T}>15 \mathrm{GeV}\right)$ or a muon $\left(P_{T}>10 \mathrm{GeV}\right)$ corresponding to an integrated luminosity of $1.98 \pm 0.02 \mathrm{fb}^{-1}$ to search for Higgs decaying into $b \bar{b}$ and $c \bar{c}$ pairs [7] produced in association with vector boson. In addition to the lepton well separated from the rest of the event, two $b$-tagged jets with momentum greater than $20 \mathrm{GeV}$ are required. The light quark jets are removed by cutting on the BDT output. After the selection requirements there is no evidence of signal, therefore limits on the production cross section times the branching ratio are set. The invariant mass of the dijet system is shown in figure 1, with both the number of expected and observed events, dominated by $W+b \bar{b}$ and $t \bar{t}$ processes. Multivariate classifiers are used to distinguish between $(W / Z) H \rightarrow b \bar{b}$ and $W+b \bar{b}$ and between $(W / Z) H \rightarrow b \bar{b}$ and $t \bar{t}$. The Confidence Levels (CLs) limit [8] at 95\%, are: $\sigma\left(p p \rightarrow Z / W+H^{0}+X\right) B F\left(H^{0} \rightarrow b \bar{b}\right)<1.6 \mathrm{pb}$ and $\sigma\left(p p \rightarrow Z / W+H^{0}+X\right) B F\left(H^{0} \rightarrow c \bar{c}\right)<9.4 \mathrm{pb}$

The limit on $H^{0} \rightarrow c \bar{c}$ is the first direct inclusive limit ever set by an experiment. In the future $\mathrm{LHCb}$ expects to improve the results by increasing the acquired luminosity and by further optimizing the jets $b$-tagging.

\section{Search for long-lived particles decaying to jet pairs}

The $\pi_{v}$ particle, pair-produced in the decay of a SM-like Higgs particle can be searched via the decay into two $b$-jet pair with a four jets final state. The two hadronic jets must originate 


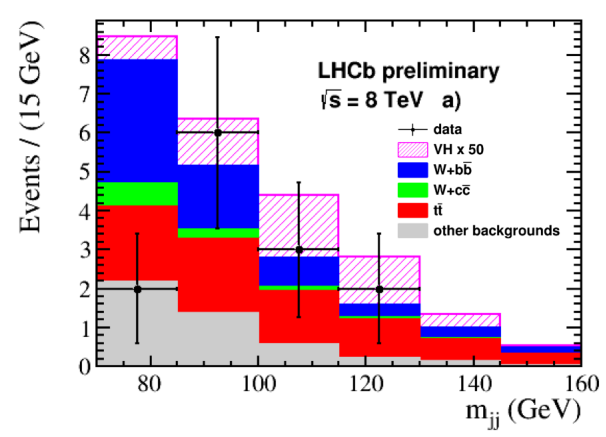

Figure 1: Di-jet invariant mass distribution of data for the muon triggered sample. The SM signal multiplied by 50 and background prediction is also shown.

from a vertex required to be radially displaced $\left(R_{x y}\right)$ from the proton-proton collision axis by more than $0.4 \mathrm{~mm}$ to reject most of the background. The analysis is performed on $0.62 \mathrm{fb}^{-1}$ at $\sqrt{\mathrm{s}}=$ $7 \mathrm{TeV}$ [9]. The $b$-jets are identified following the method already described with a parameter $R=$ 0.7 in this case. The di-jets momentum has to point back to the primary vertex and back-to-back jets are vetoed. The background yield, the shape of the background invariant mass distribution and the selection efficiency strongly depend on $R_{x y}$, therefore limits are extracted from a simultaneous maximum likelihood fit to the di-jet invariant mass distribution in bins of $R_{x y}$. The intervals are chosen in the most sensitive region, $0.4<R_{x y}<4.8 \mathrm{~mm}$. The fit procedure is performed for a $\pi_{v}$ mass of $25,35,43$ and $50 \mathrm{GeV} / \mathrm{c}^{2}$ and for several values of the lifetime between 1 and $200 \mathrm{ps}$. No significant signal is observed for any combination of $\pi_{v}$ mass and lifetime. Upper CLs limits on the Higgs production cross-section times the branching fraction into long-lived particles, are extracted by assuming both $\pi_{v}$ particles decay to the same $b \bar{b}$ final state (figure 2).

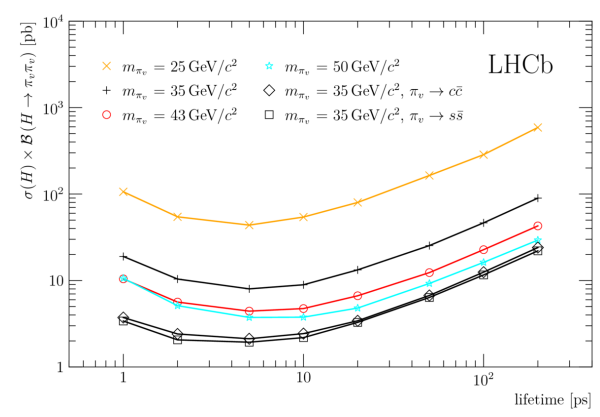

Figure 2: Observed 95\%CL cross-section upper limits on a hidden valley model for various $\pi_{v}$ masses, as a function of $\pi_{v}$ lifetime. The Higgs-like particle mass is assumed to be $120 \mathrm{GeV}$.

\section{Search for Higgs-like bosons decaying into pairs of long-lived exotic particles}

Long lived particles [10] can be produced in the decay of SUSY Higgs-like boson, $h^{0} \rightarrow \tilde{\chi}_{1}^{0} \tilde{\chi}_{1}^{0}$ with $\tilde{\chi}_{1}^{0}$ decays into 3 jets. Here, minimal supergravity model with baryon number violation of the minimal supersymmetric model is used as a benchmark model with baryon number violation. The $h^{0}$ particle mass ranges from $80 \mathrm{GeV} / c^{2}$ up to $140 \mathrm{GeV} / \mathrm{c}^{2}$. The $\tilde{\chi}_{1}^{0}$ lifetime is considered between 50 and $100 \mathrm{ps}$, longer than the typical $b$-hadron lifetime, 
corresponding to an average flight distance of up to $30 \mathrm{~cm}$, which is inside the LHCb vertex detector region. The $\tilde{\chi}_{1}^{0}$ mass range considered is from 20 to $60 \mathrm{GeV} / \mathrm{c}^{2}$. The LLP candidate is reconstructed as a secondary vertex with at least four tracks in the forward region and with a distance from the primary vertex $R_{x y}>0.4 \mathrm{~mm}$. Two LLP candidates form a Higgs-like candidate and the di-LLP invariant mass is fit to determine the signal yield. The 95\% CLs upper limits on the production cross-section times branching ratio are presented in figure 3 for data collected at $\sqrt{s}=7 \mathrm{TeV}$ corresponding to an integrated luminosity of $0.62 \mathrm{fb}^{-1}$.
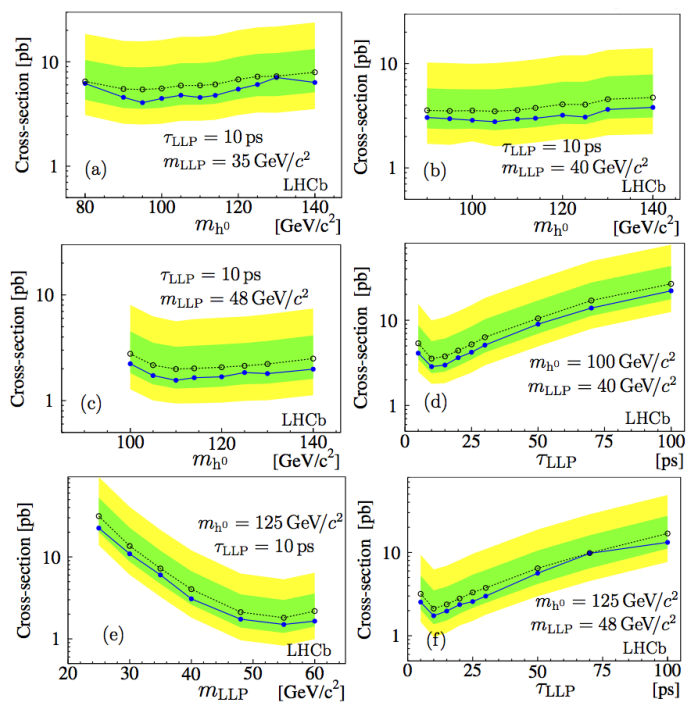

Figure 3: Expected (open dots with $1 \sigma$ and $2 \sigma$ bands) and observed (full dots) upper limits at $95 \%$ confidence level, shown for different masses of the Higgs-like particle, and for different LLP lifetimes.

\section{References}

[1] D. E. Kaplan and K. Rehermann,Proposal for Higgs and superpartner searches at the LHCb experiment, JHEP 10 (2007) 056

[2] M. J. Strassler and K. M. Zurek, Discovering the Higgs through highly-displaced vertices, Phys. Lett. B661(2008) 263.

[3] LHCb Coll., A. A. Alves Jr. et al., The LHCb detector at the LHC, JINST 3 (2008) S08005.

[4] LHCb Coll., LHCb detector performance,Int. J. Mod. Phys. A30 (2015) 1530022.

[5] LHCb Coll., Identification of beauty and charm quark jets at LHCb, JINST 10 (2015) P06013

[6] ATLAS and CMS Coll., Measurements of the Higgs boson production and decay rates and constraints on its couplings from a combined ATLAS and CMS analysis of the LHC pp collision data at $\sqrt{s}=7$ and $8 \mathrm{TeV}$, arXiv:1606.02266.

[7] LHCb Coll., Search for $H^{0} \rightarrow b \bar{b}$ or $c \bar{c}$ in association with a $W$ or $Z$ boson in the forward region of $p p$ collisions, LHCb-CONF-2016-006.

[8] A. L. Read, Presentation of search results: The CL(s) technique, J. Phys. G28 (2002) 2693.

[9] LHCb Coll., Search for long-lived particles decaying to jet pairs, Eur. J. Phys. C75 (2015) 152.

[10] LHCb Coll., Search for Higgs-like bosons decaying into long-lived exotic particles, LHCb-PAPER-2016-014, arXiv:1609.03124. 